\title{
Genetic causes of Parkinson's disease in the Maltese: a study of selected mutations in LRRK2, MTHFR, QDPR and SPR
}

\author{
Charmaine Zahra ${ }^{1}$, Christine Tabone ${ }^{2}$, Graziella Camilleri ${ }^{2}$, Alex E. Felice ${ }^{1}$, Rosienne Farrugia ${ }^{2}$ \\ and Stephanie Bezzina Wettinger ${ }^{1,2^{*}}$
}

\begin{abstract}
Background: Mutations in Leucine-rich repeat kinase 2 NM_198578 (LRRK2 c.6055G > A (p.G2019S), LRRK2 c. 4321C > G (p.R1441G)) and alpha-synuclein NM_000345 (SNCA c.209G > A (p.A53T)) genes causing Parkinson's disease (PD) are common in Mediterranean populations. Variants in the Quinoid Dihydropteridine Reductase NM_000320 (QDPR c.68G > A (p.G23D)), Sepiapterin Reductase NM_003124 (SPR c.596-2A > G) and Methylenetetrahydrofolate Reductase NM_005957 (MTHFR c.677C > T and c.1298A > C) genes are frequent in Malta and potential candidates for PD.
\end{abstract}

Methods: 178 cases and 402 control samples from Malta collected as part of the Geoparkinson project were genotyped for MTHFR polymorphisms, QDPR and SPR mutations. Only PD and parkinsonism cases were tested for SNCA and LRRK2 mutations.

Results: LRRK2 c.4321C > G and SNCA c.209G > A were not detected. The LRRK2 c.6055G > A mutation was found in $3.1 \%$ of Maltese PD cases. The QDPR mutation was found in both cases and controls and did not increase risk for PD. The SPR mutation was found in controls only. The odds ratios for MTHFR polymorphisms were not elevated.

Conclusions: The LRRK2 c.6055G > A is a cause of PD in the Maltese, whilst QDPR c.68G > A, SPR c.596-2A > G and MTHFR c.677C > T and C.1298A > C are not important determinants of PD.

Keywords: Quinoid Dihydropteridine Reductase, Sepiapterin Reductase, Methylenetetrahydrofolate Reductase, Leucine-rich repeat kinase 2, Alpha-synuclein, Parkinson's Disease, Maltese

\section{Background}

Parkinson's disease (PD) is a neurodegenerative disorder characterised by motor and non-motor symptoms and loss of dopaminergic neurons. A number of candidate genes were investigated to uncover the PD causing mutations in the Maltese population. Mutations in leucine-rich repeat kinase 2 NM_198578 (LRRK2) and alpha-synuclein NM_000345 (SNCA) genes have been associated with $\mathrm{PD}$. Three mutations in these genes, namely $L R R K 2$ c.6055G > A, LRRK2 c.4321C > G and SNCA c.209G > A

\footnotetext{
* Correspondence: stephanie.bezzina-wettinger@um.edu.mt ${ }^{1}$ Laboratory of Molecular Genetics, Department of Physiology and Biochemistry, University of Malta, Msida, Malta

${ }^{2}$ Department of Applied Biomedical Science, Faculty of Health Sciences,

University of Malta, Msida, Malta
}

(c) 2016 The Author(s). Open Access This article is distributed under the terms of the Creative Commons Attribution 4.0 International License (http://creativecommons.org/licenses/by/4.0/), which permits unrestricted use, distribution, and reproduction in any medium, provided you give appropriate credit to the original author(s) and the source, provide a link to the Creative Commons license, and indicate if changes were made. The Creative Commons Public Domain Dedication waiver (http://creativecommons.org/publicdomain/zero/1.0/) applies to the data made available in this article, unless otherwise stated.

are pathogenic mutations found in Mediterranean populations [1]. The Quinoid Dihydropteridine Reductase NM_000320 (QDPR) c.68G > A mutation and Sepiapterin Reductase NM_003124 (SPR) c.596-2A > G splice variant, are autosomal recessive mutations known to cause dihydropteridine reductase (DHPR) deficiency and Dopa-Responsive Dystonia (DRD) respectively in the Maltese with a high carrier frequency compared to other populations [2]. Catecholamine synthesis in patients is impaired as tetrahydrobiopterin $\left(\mathrm{BH}_{4}\right)$, an essential cofactor of aromatic amino acid hydroxylases, is not produced in sufficient amounts as a result of these mutations [3]. SR is involved in de novo $\mathrm{BH}_{4}$ synthesis whilst DHPR is involved in recycling of the reduced cofactor [4]. It has been suggested that the SPR gene is 
the PARK3 locus implicated in sporadic PD [1] and decreased activity of DHPR due to low biopterin levels causes PD [5]. DHPR deficiency and DRD and PD have symptoms in common. The latter is characterized by childhood-onset dystonia responding well to low doses of L-Dopa. Gait and posture abnormalities are observed as the legs are primarily affected with diurnal fluctuation. Symptoms of parkinsonism (PS) are common. DHPR deficiency also presents with tonic disorders, abnormal movements, psychomotor retardation, hypersalivation and drooling, swallowing difficulties, hyperthermia $[3,6]$. There is evidence that both the SPR and QDPR genes are good candidates to test for associations for PD.

MTHFR reduces folate and is involved in the production of S-adenosylmethionine which plays a role in dopamine (DA) synthesis and metabolism [7]. Defective MTHFR enzyme due to MTHFR NM_005957 c.1298A > C and c.677C $>\mathrm{T}$ has a lower activity and may result in elevated homocysteine levels, found to be relatively high in sera of PD patients [7, 8]. An indirect connection between the $\mathrm{BH}_{4}$ and folate pathways has been postulated [9]. The MTHFR c.677C > T TT genotype is found at a frequency of $9.1 \%$ in Maltese newborn [10]. The mutant allele frequency for the MTHFR c.1298A > C was reported to be $23.2 \%[11]$.

The objective of this study was to establish whether known pathogenic mutations (LRRK2 c.6055G > A, LRRK2 c.4321C > G and SNCA c.209G > A) are present in Maltese $\mathrm{PD}$ and PS cases, and to determine whether the functional MTHFR c.677C > T and c.1298A > C, QDPR c.68G > A and $S P R$ c.596-2A $>$ G genetic variants alter susceptibility to $\mathrm{PD}$. This is the first time these genes have been tested for involvement in PD in the Maltese population.

\section{Methods}

Altogether 178 cases and 402 controls (Table 1) from Malta were collected as part of the FP5 EU-funded Geoparkinson project $[12,13]$. The collection and testing were approved by the Research Ethics Committee of the University of Malta, Approval Numbers 53/2002 and 52/2008. All participants gave written informed consent.

Cases were classified as having PD or PS using the United Kingdom Parkinson's Disease Society Brain Bank clinical diagnostic criteria. Controls did not have PD or PS and were recruited from the community and from the bleeding room at St Luke's Hospital which served many outpatient departments.

Genotyping of the QDPR c.68G > A, SPR c.596-2A > G [2], MTHFR c.677C > T [14], LRRK2 c.6055G > A [15], LRRK2 c.4321C > G [16] and SNCA c.209G > A [17] was performed as described previously. Genotyping of the MTHFR c.1298A > C was done using the 5' exonuclease assay [18]. Cases and controls were genotyped for the QDPR c.68G > A, SPR c.596-2A > G mutations and the
Table 1 Characteristics of the population studied

\begin{tabular}{lll}
\hline Characteristics & $\begin{array}{l}\text { Cases } \\
(N=178)\end{array}$ & $\begin{array}{l}\text { Controls } \\
(N=402)\end{array}$ \\
\hline Mean age (range) & $72.9(41-91)$ & $74.3(41-95)$ \\
Mean age of diagnosis ${ }^{\mathrm{a}}$ & $66.3(35-90)$ & \\
Males & $110(61.8 \%)$ & $224(55.7 \%)$ \\
Females & $68(39.2 \%)$ & $178(44.3 \%)$ \\
Parkinson's Disease & 118 & \\
Parkinsonism & 60 & \\
\% Parkinsonism & $33.70 \%$ & \\
Smokers & \\
Ever knocked unconscious $^{\mathrm{e}}$ & $61(34.3 \%)$ & $177(44.0 \%)$ \\
Family history of Parkinson's disease $^{\mathrm{e}}$ & $33^{\mathrm{b}}(18.6 \%)$ & $48(11.9 \%)$ \\
Use of pesticides $^{\mathrm{e}}$ & $35(19.7 \%)$ & $16^{\mathrm{c}}(4.0 \%)$ \\
\hline
\end{tabular}

${ }^{a}$ Out of 135 cases for which this data was available

bOut of 177 cases

'Out of 401 controls

${ }^{\mathrm{d}}$ Associated with decreased risk for PD and PS

${ }^{\mathrm{e}}$ Associated with increased risk for PD and PS

MTHFR c. $677 \mathrm{C}>\mathrm{T}$ and c.1298A $>\mathrm{C}$ polymorphisms. Genotyping for the LRRK2 c.6055G > A, LRRK2 c.4321C > G and SNCA c.209G > A was limited to cases since these are known PD-causing mutations [1].

DNA of 79 controls and 27 cases collected as part of the Geoparkinson project had been used up. These were mainly buccal samples. Additionally the PCRs did not work in a few samples.

Data Analysis: Allele frequencies were calculated. The genotypes in controls, when available, were tested for conformity with the Hardy-Weinberg Equilibrium (HWE). Both the crude and adjusted odds ratios (ORs) were calculated and the $95 \%$ confidence intervals $(\mathrm{CI})$ were derived from the logistic regression model.

\section{Results}

Four cases were heterozygous for the $L R R K 2$ c.6055G > A mutation, giving an allele frequency of $1.4 \%$ in $\mathrm{PD}$ and PS cases. This frequency was $3.1 \%$ in PD cases alone. All were males with no family history of disease. Data for age at diagnosis was only available for two cases, one with PD and one with PS, both diagnosed at 65 years of age. None of the cases had the LRRK2 c. $4321 \mathrm{C}>\mathrm{G}$ or $S N C A$ c.209G > A mutations. The frequencies obtained for the variants studied are presented in Tables 2 and 3.

Odds ratios for combined PD and PS cases were OR 0.9 (95 \% CI 0.5-1.6) for the MTHFR 677 TT and 1.2 (95\% CI 0.6-2.1) for the MTHFR 1298 CC genotypes respectively. The results did not change materially after restricting to cases with PD or PS (see Additional file 1). No deviations from HWE were observed. 
Table 2 Allele Frequencies for the variants studied

\begin{tabular}{lll}
\hline Mutation/polymorphism & \multicolumn{2}{l}{ Allele frequencies in \% } \\
\cline { 2 - 3 } & $\begin{array}{l}\text { Controls } \\
(\mathrm{n} \text { mutant/total } \mathrm{n})^{\mathrm{a}}\end{array}$ & $\begin{array}{l}\text { Cases } \\
(\mathrm{n} \text { mutant/total } \mathrm{n})^{\mathrm{a}}\end{array}$ \\
\hline QDPR c.68G > A & $0.3(2 / 612)$ & $0.3(1 / 300)$ \\
SPR c.596-2A > G & $0.7(4 / 594)$ & $0.0(0 / 296)$ \\
MTHFR c.677C > T & $34.7(216 / 622)$ & $33.8(102 / 302)$ \\
MTHFR c.1298A > C & $35.9(225 / 626)$ & $37.7(114 / 302)$ \\
Tested in cases only & & \\
LRRK2 c.6055G > A & - & $1.4(4 / 292)$ \\
LRRK2 c.4321C > G & - & $0.0(0 / 296)$ \\
SNCA c.209G > A & - & $0.0(0 / 292)$
\end{tabular}

$n=$ number of alleles

${ }^{a}$ DNA of 79 controls and 27 cases had been used up and were therefore not available and a few samples did not yield PCR products

The allele frequency for $Q D P R$ c.68G > A was $0.3 \%$ in both cases and controls. Two controls and one case were heterozygous for QDPR c.68G > A. One control was a 73 year old male whilst the other control was a 66 year old female. The case identified fulfilled the criteria for PD. She was 53 years of age when diagnosed and was also heterozygous for the MTHFR c.1298A > C polymorphism. None had a family history of PD.

Four individuals were identified as carriers of SPR c.596-2A > G, all of which were controls giving an allele frequency of $0.7 \%$. One was female aged 70 , whilst the others were all men with ages of 79, 49 and 66 .

\section{Discussion}

The known disease-causing $L R R K 2$ c.6055G > A mutation was detected in PD cases whereas the $L R R K 2$ c. $4321 \mathrm{C}>\mathrm{G}$ and SNCA c.209G > A mutations were not detected. The allele frequency of $Q D P R$ c.68G > A was the same in the cases and the controls whilst $S P R$ c.596-2A > G was not detected in cases. The MTHFR c.677C $>\mathrm{T}$ and MTHFR c.1298A $>\mathrm{C}$ polymorphisms were not associated with $\mathrm{PD}$ in this study.

Four cases were heterozygous for the LRRK2 c.6055G > A mutation giving an allele frequency of $1.4 \%$ in PD and PS cases or $3.1 \%$ in PD cases alone. LRRK2 (c.4321C > G and c.6055G > A) mutations cause amino acid changes within the GTPase and/or kinase domains respectively which may signify an enzymatic change and thus mediate $L R R K 2$ pathogenesis $[19,20]$. The frequency of $L R R K 2$ c.6055G > A was estimated to be $1 \%$ of patients with sporadic PD and $4 \%$ of patients with hereditary PD in other populations, with the highest frequencies occurring in Arabs and Jews but is rare in Asia [21]. This may be explained by differences in descent and founder effects [22]. The frequency of this mutation in Maltese PD patients, $3.1 \%$, is similar to that in the Spanish, Italians and Sardinians [21]. This is the first report of this mutation being found in the Maltese.

The LRRK2 c. $4321 \mathrm{C}>\mathrm{G}$ is common in the Basque population [23, 24]. The $S N C A$ c.209G $>$ A mutation is thought to have arisen from a common founder with Mediterranean ancestry $[25,26]$. The results of the present study indicate that the $L R R K 2$ c. $4321 \mathrm{C}>\mathrm{G}$ and

Table 3 Genotype Frequencies for MTHFR, all cases (PD \& PS)

\begin{tabular}{|c|c|c|c|c|c|}
\hline \multicolumn{2}{|l|}{ Polymorphism } & \multicolumn{3}{|c|}{ Genotype frequencies } & \multirow[t]{2}{*}{$p$-value } \\
\hline MTHFR c.677C > T & MTHFR C.1298A >C & No. of Cases $(\%)^{a}$ & No. of Controls (\%) ${ }^{a}$ & Age-adjusted OR $(95 \% \mathrm{Cl})^{\mathrm{b}}$ & \\
\hline CC & & $66(43.7)$ & $136(43.7)$ & 1 & \\
\hline$C T$ & & $68(45.0)$ & $134(43.1)$ & $1.0(0.7-1.6)$ & 0.8 \\
\hline \multirow[t]{4}{*}{$\pi$} & & $17(11.3)$ & $41(13.2)$ & $0.9(0.5-1.6)$ & 0.6 \\
\hline & AA & $60(39.7)$ & $132(42.2)$ & 1 & \\
\hline & $A C$ & $68(45.0)$ & $137(43.8)$ & $1.1(0.7-1.7)$ & 0.7 \\
\hline & CC & $23(15.2)$ & $44(14.1)$ & $1.2(0.6-2.1)$ & 0.6 \\
\hline CC & $\mathrm{AA}$ & $16(10.6)$ & $24(7.7)$ & 1 & \\
\hline$C T$ & $A C$ & $41(27.2)$ & $67(21.6)$ & $0.9(0.4-2.0)$ & 0.8 \\
\hline$\pi$ & CC & - & - & - & \\
\hline CC & $A C$ & $27(17.9)$ & $69(22.3)$ & $0.6(0.3-1.3)$ & 0.2 \\
\hline CC & CC & $23(15.2)$ & $43(13.9)$ & $0.8(0.4-1.8)$ & 0.6 \\
\hline$C T$ & AA & $27(17.9)$ & $66(21.3)$ & $0.6(0.3-1.3)$ & 0.2 \\
\hline$C T$ & CC & - & - & - & \\
\hline$\pi$ & $\mathrm{AA}$ & $17(11.3)$ & $41(13.2)$ & $0.6(0.3-1.5)$ & 0.3 \\
\hline$T$ & $A C$ & - & - & - & \\
\hline
\end{tabular}

aNA of 79 controls and 27 cases had been used up and were therefore not available and a few samples did not yield PCR products ${ }^{b} p$-values were all $>0.05$ 
SNCA c.209G > A mutations are very rare or completely absent in the Maltese population.

The allele frequencies for the $Q D P R$ c.68G $>\mathrm{A}$ and SPR c.596-2A > G we observed in controls $(0.3 \%$ and $0.7 \%$ respectively) are much lower than those reported previously (3.3\% and $4.6 \%$ tested on 272 and 87 newborns respectively) [2]. Therefore, we tested the population frequency in a larger number of Maltese newborn and observed allele frequencies of $0.64 \%$, and $1.3 \%$ for the QDPR and SPR polymorphisms respectively ( $N=475$ and 315 respectively). These mutations are not a major cause of PD in the Maltese.

MTHFR was not a contributor to PD. The genotype frequencies obtained for the MTHFR c.677C $>\mathrm{T}$ are 43.7 \% CC, $43.1 \%$ CT and $13.2 \%$ TT which are comparable to others reported for Maltese newborn [10, 27]. In contrast, the mutant allele frequency for the MTHFR c.1298A > C was somewhat higher than that observed previously in Maltese newborn (35.9 \% vs. $23.2 \%$ [11]). This suggests that there is no clear effect of these SNPs on PD probably due to the complex pathways involved.

The c.677C $>\mathrm{T}$ and c.1298A $>\mathrm{C}$ prevalence and frequencies vary considerably with ethnicities and geography [7]. While some studies have reported a lack of association between MTHFR polymorphisms and PD [28], others [29] reported a higher frequency of the MTHFR 677 TT genotype in PD patients and controls.

The MTHFR c.667C > T dimorphism causes hyperhomocysteimia in folate deficient individuals [30]. Homozygous mutants for MTHFR c.1298A > C have a lower enzyme activity than homozygous wildtype [7]. It is possible that the Maltese diet includes sufficient folate levels. In fact only $1.5 \%$ of adult Maltese women and $3.1 \%$ of adult Maltese men had low serum folate levels in a group of population controls and $1.5 \%$ of women and $1.2 \%$ of men had low serum Vitamin B12 levels ( $N=137$ and $N=326$ for women and men respectively; Attard R and Bezzina Wettinger S, unpublished observations). The chance of being heterozygous or homozygous for these polymorphisms with low folate status is therefore very low. It should be noted that higher nutrient intake (folate and B vitamins) did not lower PD risk in a previous study [31]. It cannot be excluded that these polymorphisms have an effect in populations or individuals with low folate and Vitamin B12 levels.

This study had $80 \%$ power to detect a 2 -fold increase in risk for the MTHFR polymorphisms (35\% allele frequency), a 5-fold increased risk for SPR c.596-2A > G $(0.7 \%$ allele frequency) and an 8 -fold increased risk for QDPR c.68G > A (0.3 \% allele frequency) assuming a dominant model and for the MTHFR polymorphisms the sample size was sufficient to detect a two-fold increase in risk even for a recessive model assuming up to a $5 \%$ Type I error rate. Thus the study is sufficiently powered to exclude a strong effect on PD risk of the $S P R$ and QDPR mutations, and even 2-fold or more increase in risk due to the MTHFR mutations.

\section{Conclusion}

The LRRK2 c.6055G > A mutation accounts for $3.1 \%$ of PD in the Maltese population. As LRRK2 c.6055G > A exhibits low penetrance and is found occasionally in controls [21], testing for $L R R K 2$ c.6055G > A may be performed on patients and relatives who wish to ascertain the presence of a genetic defect. Testing for LRRK2 c.4321C > G and SNCA c.209G > A are not recommended as a first line of genetic testing in Maltese PD patients. There is no evidence to support involvement of DHPR and SR in PD, at least alone. One cannot exclude that they have a weak effect and even possibly an interaction when found together with other mild mutations. The absence of the LRRK2 c. $4321 \mathrm{C}>\mathrm{G}$ and SNCA c.209G > A could be a result of historical bottlenecks, and there may be some founder mutation in the Maltese that may explain familial cases that has not yet been tested for.

\section{Additional file}

Additional file 1: Genotype frequencies for MTHFR, Parkinson's Disease (Table S1a) and Parkinsonism (Table S1b) only. (DOC 57.5 kb)

\section{Abbreviations}

$\mathrm{BH}_{4}$ : Tetrahydrobiopterin; Cl: Confidence intervals; DA: Dopamine; DHPR: Dihydropteridine reductase; DRD: Dopa-Responsive Dystonia; HWE: Hardy-Weinberg Equilibrium; L-Dopa: Levodopa; LRRK2: Leucine-rich repeat kinase 2; MTHFR: Methylenetetrahydrofolate Reductase; OR: Odds ratios; PD: Parkinson's disease; PS: Parkinsonism; QDPR: Quinoid Dihydropteridine Reductase; SNCA: Alpha-synuclein; SNP: Single Nucleotide Polymorphism; SPR: Sepiapterin Reductase

\section{Acknowledgments}

The samples and data used in this study were collected as part of the 5th framework (FP5) EU funded Geoparkinson study, project number QLK4-CT1999-01133. The Maltese arm of this group included Prof Christian Scerri, Dr Joseph Borg, Dr Karen Cassar, Ms Wilma Cassar, Ms Ruth Galdies, Dr Norbert Vella, Dr Vicky Mifsud, Dr Josanne Aquilina and Dr Galea Debono.

\section{Funding}

The samples and data used in this study were collected as part of the 5th framework (FP5) EU funded Geoparkinson study, project number QLK4-CT1999-01133. This work was supported by research grants of SBW and RF from the University of Malta. The funders played no other part in the research or its interpretation.

\section{Availability of data and material}

Complete crosstabs were included to allow replication of calculations. Any further information can be made available on request to the corresponding author.

\section{Authors' contributions}

CZ was responsible for the execution of the research project, performed the statistical analysis and writing of the first draft. CT was responsible for the execution and analysis of the QDPR population testing. GC was responsible for the execution and analysis of the SPR testing. AEF was a reviewer and critique of the research project and the manuscript. RF designed and analyzed the research project concerning the newborn population studies. SBW designed, organized, reviewed and critically analyzed the research 
project and statistical analysis and was a reviewer and critique of the manuscript. All authors read and approved the final manuscript.

\section{Competing interests}

The authors declare that they have no competing interests.

\section{Consent for publication}

Not applicable.

\section{Ethics approval and consent to participate}

The collection and testing were approved by the Research Ethics Committee of the University of Malta, Approval Numbers 53/2002 and 52/2008. All participants gave written informed consent.

\section{Received: 4 July 2015 Accepted: 27 August 2016}

\section{Published online: 09 September 2016}

\section{References}

1. Gasser T. Mendelian forms of Parkinson's disease. Biochim Biophys Acta. 2009:1792(7):587-96.

2. Farrugia R, Scerri CA, Montalto SA, Parascandolo R, Neville BGR, Felice AE. Molecular genetics of tetrahydrobiopterin (BH4) deficiency in the Maltese population. Mol Genet Metab. 2007;90(3):277-83

3. Blau N, Thony B, Cotton RGH, Hyland K. Disorders of tetrahydrobiopterin and related biogenic amines. In: Scriver CR, Beaudet AL, Sly WS, Valle D, Childs B, Vogelstein B, Eds. The metabolic and molecular bases of inherited disease. 8th ed. New York: McGraw-Hill; 2001. p. 1275-776.

4. Thöny B. Tetrahydrobiopterin and its function. In: Blau N, editor. PKU and $\mathrm{BH} 4$ : Advances in Phenylketonuria and Tetrahydrobiopterin Research. Heilbronn: SPS Publications; 2006. p. 503-54.

5. Fujishiro K, Hagihara M, Takahashi A, Nagatsu T. Concentrations of neopterin and biopterin in the cerebrospinal fluid of patients with Parkinson's disease. Biochem Med Metab Biol. 1990:44(2):97-100.

6. Neville BG, Parascandalo R, Farrugia R, Felice A. Sepiapterin reductase deficiency: a congenital dopa-responsive motor and cognitive disorder. Brain. 2005;128(Pt 10):2291-6.

7. Botto LD, Yang Q. 5,10-Methylenetetrahydrofolate reductase gene variants and congenital anomalies: a HuGE review. Am J Epidemiol. 2000;151(9):862-77.

8. Kuhn W, Roebroek R, Blom H, et al. Elevated plasma levels of homocysteine in Parkinson's disease. Eur Neurol. 1998:40(4):225-7.

9. Kaufman S. Some metabolic relationships between biopterin and folate: implications for the "methyl trap hypothesis". Neurochem Res. 1991;16(9):1031-6.

10. Bezzina Wettinger S, Balim Z, Felice A. Allele frequencies of selected polymorphisms related to thrombosis in the Maltese population. Abstract no 4089 Abstracts for the 42nd annual meeting of American society of Heamatology Blood 96 Part 2 94b. 2000.

11. Al-Ashtar, A.A. A, J., Bezzina Wettinger $\mathrm{S}$, et al. Case-control study of Libyan and Maltese Patients with Type II Diabetes Mellitus. Eur J Human Genet. 2007;15(5) (Suppl.1). P1148.

12. Dick FD, De Palma G, Ahmadi A, et al. Gene-environment interactions in parkinsonism and Parkinson's disease: the Geoparkinson study. Occup Environ Med. 2007;64(10):673-80

13. Dick FD, De Palma G, Ahmadi A, et al. Environmental risk factors for Parkinson's disease and parkinsonism: the Geoparkinson study. Occup Environ Med. 2007;64(10):666-72.

14. Skibola CF, Smith MT, Kane E, et al. Polymorphisms in the methylenetetrahydrofolate reductase gene are associated with susceptibility to acute leukemia in adults. Proc Natl Acad Sci U S A. 1999:96(22):12810-5.

15. Punia S, Behari M, Govindappa ST, et al. Absence/rarity of commonly reported LRRK2 mutations in Indian Parkinson's disease patients. Neuroscilett. 2006; 409(2):83-8.

16. Paisan-Ruiz C, Jain S, Evans EW, et al. Cloning of the gene containing mutations that cause PARK8-linked Parkinson's disease. Neuron. 2004; 44(4):595-600

17. Polymeropoulos $M H$, Lavedan C, Leroy $\mathrm{E}$, et al. Mutation in the alphasynuclein gene identified in families with Parkinson's disease. Science. 1997; 276(5321):2045-7.

18. Rudd MF, Sellick GS, Allinson R, Matutes E, Catovsky D, Houlston RS. MTHFR polymorphisms and risk of chronic lymphocytic leukemia. Cancer Epidemiol Biomarkers Prev. 2004;13(12):2268-70.
19. Gomez-Suaga P, Fdez E, Blanca Ramirez M, Hilfiker S. A link between autophagy and the pathophysiology of LRRK2 in Parkinson's disease. Parkinsons Dis. 2012;2012:324521.

20. Kumari U, Tan EK. LRRK2 in Parkinson's disease: genetic and clinical studies from patients. FEBS J. 2009;276:6455-63.

21. Healy DG, Falchi M, O'Sullivan SS, et al. Phenotype, genotype, and worldwide genetic penetrance of LRRK2-associated Parkinson's disease: a case-control study. Lancet Neurol. 2008;7(7):583-90.

22. Zabetian CP, Hutter CM, Yearout D, et al. LRRK2 G2019S in families with Parkinson disease who originated from Europe and the Middle East: evidence of two distinct founding events beginning two millennia ago. Am J Hum Genet. 2006;79(4):752-8.

23. Mata IF, Taylor JP, Kachergus J, et al. LRRK2 R1441G in Spanish patients with Parkinson's disease. NeurosciLett. 2005;382(3):309-11.

24. Spira PJ, Sharpe DM, Halliday G, Cavanagh J, Nicholson GA. Clinical and pathological features of a Parkinsonian syndrome in a family with an Ala53Thr alpha-synuclein mutation. Ann Neurol. 2001:49(3):313-9.

25. Markopoulou K, Wszolek ZK, Pfeiffer RF, Chase BA. Reduced expression of the G209A alpha-synuclein allele in familial Parkinsonism. AnnNeurol. 1999; 46(3):374-81.

26. Berg D, Niwar M, Maass S, et al. Alpha-synuclein and Parkinson's disease: implications from the screening of more than 1,900 patients. Mov Disord. 2005;20(9):1191-4

27. Vidal C, Brincat $M$, Xuereb-Anastasi A. Effects of polymorphisms in the collagen type 1 a1 gene promoter and the C677T variant in the methylenetetrahydrofolate reductase gene on bone mineral density in postmenopausal women in Malta. Balkan J Med Genet. 2007;10:9-18.

28. Religa D, Czyzewski K, Styczynska M, et al. Hyperhomocysteinemia and methylenetetrahydrofolate reductase polymorphism in patients with Parkinson's disease. NeurosciLett. 2006;404(1-2):56-60.

29. Todorovic Z, Dzoljic E, Novakovic l, et al. Homocysteine serum levels and MTHFR C677T genotype in patients with Parkinson's disease, with and without levodopa therapy. JNeurolSci. 2006;248(1-2):56-61.

30. Jacques PF, Bostom AG, Williams RR, et al. Relation Between Folate Status, a Common Mutation in Methylenetetrahydrofolate Reductase, and Plasma Homocysteine Concentrations. Circulation. 1996;93:7-9.

31. Chen $\mathrm{H}$, Zhang SM, Schwarzschild MA, et al. Folate intake and risk of Parkinson's disease. Am J Epidemiol. 2004;160(4):368-75.

\section{Submit your next manuscript to BioMed Central and we will help you at every step:}

- We accept pre-submission inquiries

- Our selector tool helps you to find the most relevant journal

- We provide round the clock customer support

- Convenient online submission

- Thorough peer review

- Inclusion in PubMed and all major indexing services

- Maximum visibility for your research

Submit your manuscript at www.biomedcentral.com/submit
C) Biomed Central 